\title{
Early and Late Histological and Ultrastructural Findings in Resected Infantile Capillary Hemangiomas Following Treatment with Topical Beta-Blocker Timolol Maleate $\mathbf{0 . 5 \%}$
}

\author{
Zuzana Sipkova $^{a}$ Kanmin Xue ${ }^{a, b}$ Hardeep S. Mudhar ${ }^{d}$ Bart Wagner $^{\mathrm{e}}$ \\ Göran Darius Hildebrand ${ }^{\mathrm{a}, \mathrm{c}}$ \\ ${ }^{a}$ Oxford Eye Hospital, Oxford University Hospital NHS Foundation Trust, ${ }^{b}$ Nuffield Laboratory of Ophthalmology, \\ Nuffield Department of Clinical Neurosciences, University of Oxford, and ' Oxford Children's Hospital, Oxford \\ University Hospital NHS Foundation Trust, Oxford, ${ }^{\circledR}$ National Specialist Ophthalmic Pathology Service (NSOPS), \\ Department of Histopathology, Royal Hallamshire Hospital, and eElectron Microscopy Unit, Department of \\ Histopathology, Royal Hallamshire Hospital, Sheffield, UK
}

\section{Keywords}

Infantile capillary hemangioma $\cdot$ Topical $\beta$-blocker $\cdot$ Topical timolol maleate $0.5 \%$

\begin{abstract}
Background: Infantile capillary hemangiomas $(\mathrm{IHs})$ affect approximately $4-5 \%$ of infants. The systemic nonselective $\beta$-adrenergic antagonist, propranolol, has become the standard first-line treatment for severe IHs. The topical $\beta$-antagonist, timolol maleate, has also demonstrated efficacy and safety in treating superficial and some deep capillary hemangiomas. Despite their therapeutic success and prevalent use, the mechanism of action of $\beta$-adrenergic antagonists in the treatment of $\mathrm{IHs}$ is not well understood. Methods: Histopathological and electron microscopic evaluation of two periocular IHs excised at 1 week and 24 months following topical timolol treatment was performed. Results: Distinct morphological differences were observed between spontaneously regressed and $\beta$-antagonist-treated IHs. The
\end{abstract}

(c) 2017 S. Karger AG, Basel

E-Mail karger@karger.com www.karger.com/oop former was characterized by diffuse collagen deposition and interstitial fibrosis, while the latter showed organized concentric collagen IV deposition within obliterated vessel lumen, suggestive of waves of endothelial cell apoptosis, leaving behind layers of basement membrane deposits as a stress response. Conclusions: Based on these observations, we hypothesize that, apart from their well-known cardiac and vasodilatory effects, $\beta$-antagonists could induce endothelial cell apoptosis in IH leading to endovascular occlusion and we present supporting evidence to explain why this response might be specific to hypoxic tissue.

(c) 2017 S. Karger AG, Basel

\section{Background}

Infantile capillary hemangioma (IH) is the most common benign tumor of childhood, affecting approximately $4-5 \%$ of infants, and up to a third of premature infants [1]. IHs are characterized by an initial proliferative phase 
(from birth up to the age of 1 year) followed by slow spontaneous involution lasting up to 10 years. While benign, IH may cause disfigurement and other serious complications. Periocular IH may lead to rapid onset of vision loss from amblyopia due to secondary astigmatism, anisometropia, and strabismus and, therefore, may require immediate intervention to prevent permanent sight loss.

Since the serendipitous observation of efficacy of the nonselective $\beta_{1} / \beta_{2}$-adrenergic antagonist propranolol in treating $\mathrm{IH}$, significant advances have been made [2]. The clinical use of $\beta$-antagonists either orally or topically has been shown to be efficacious in inducing regression of $\mathrm{IH}$ [3-6]. Topical timolol maleate, a nonselective $\beta$-antagonist with a lipophilic moiety that enhances transdermal absorption, induces regression of periocular IH with minimal side effects [3-6]. It is increasingly considered an alternative option to oral propanolol not only for superficial, but also deep periocular IHs [5-7].

Despite their therapeutic success, the mechanism of action of $\beta$-adrenergic antagonists in the treatment of $\mathrm{IH}$ is not well understood. We describe the first histopathological and transmission electron microscopy observations from treated periocular IHs excised at 1 week and 24 months following initiation of topical timolol maleate therapy. The findings provide novel insights into the mechanisms of action of $\beta$-adrenergic antagonists in the treatment of $\mathrm{IH}$.

\section{Case Report}

Case 1

A 10-week-old Caucasian boy presented with a left anterior orbital lesion above the medial canthus, causing lateral displacement of the globe and mild ptosis (Fig. 1a). It was first noticed by the parents at 3 weeks of age and had been growing rapidly ever since. No significant birth, general medical and family history was given. Ocular examination was otherwise normal. MRI of the orbits was in keeping with an infantile hemangioma. An initial trial of topical timolol maleate $0.5 \%$, applied by rubbing 3 drops twice daily into the skin overlying the lesion, was commenced, as described previously [4-6], but no significant effect was seen after a week. Therefore, an urgent diagnostic orbital biopsy was performed (Fig. 1b). Hematoxylin and eosin ( $\mathrm{H} \& \mathrm{E})$ staining confirmed a typical capillary hemangioma in its active proliferative phase with back-toback capillary-sized vessels, some of which lacked lumens (Fig. 2a). Immunohistochemistry of the lesion demonstrated the presence of CD31- (Fig. 2b), CD34- and GLUT-1-positive (not shown) endothelial cells, surrounded by smooth muscle actin (SMA)-positive pericytes (Fig. 2c). Conspicuous apoptotic bodies were seen at the level of the endothelial cells (Fig. 2d-f), a feature that was not observed in an untreated capillary hemangioma (not shown). Transmission electron microscopy (TEM) confirmed endothelial cell apoptosis (Fig. 2g).

Histopathology of Topical Beta-BlockerTreated Capillary Hemangioma

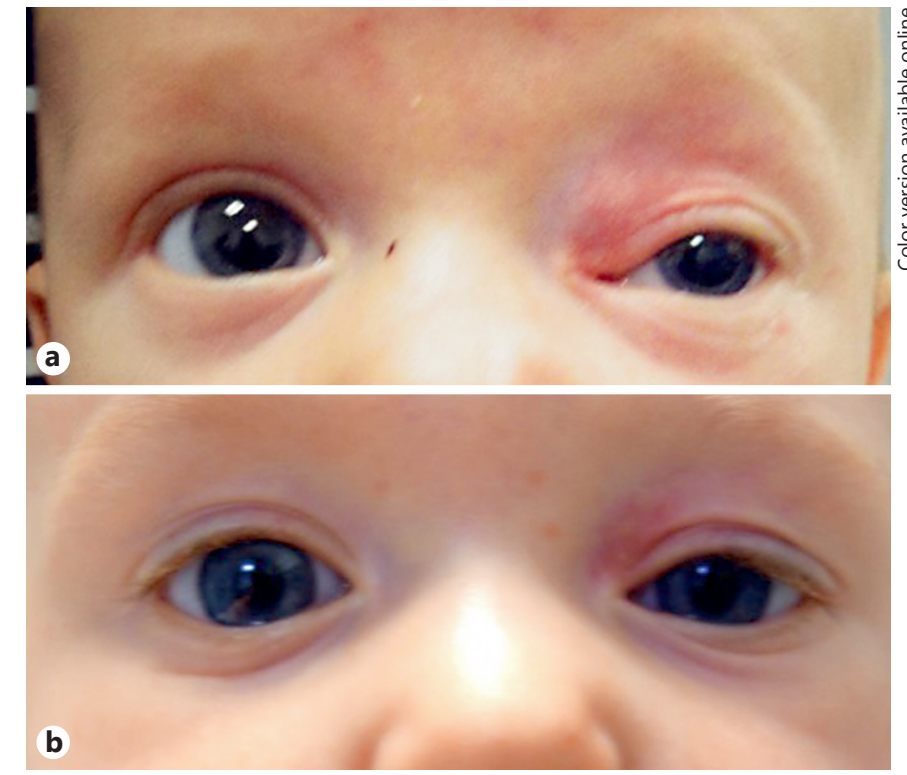

Fig. 1. Photographs of case 1. a A deep infantile capillary hemangioma of the superonasal aspect of the left anterior orbit in a 10 -week-old boy prior to treatment. b Postoperative appearance after orbital biopsy of the lesion, which was performed after 1 week of topical timolol maleate treatment with limited response.

Case 2

An 8-month-old Caucasian girl presented with a large superficial IH involving the right upper lid (Fig. 3a). This 'strawberry nevus' was first noted by the parents a few weeks after birth and had rapidly increased in size until the age of 4 months, when it stopped growing. Birth, and medical and family history were unremarkable. No orbital involvement was evident with full extraocular movements, no strabismus and no proptosis. Although the infant appeared to object equally to occlusion of either eye, indicative of symmetrical visual acuity, she was adopting an abnormal head posture with chin elevation to minimize partial encroachment of the visual axis by the overhanging upper lid $\mathrm{IH}$.

Topical timolol maleate $0.5 \%, 3$ drops applied twice daily to the skin overlying the $\mathrm{IH}$, was commenced in the same way as described above. Blanching and softening of the lesion was seen within a week, followed by progressive involution over 24 months of topical timolol treatment with no reported side effect. After the involution of the IH mass, the residual excess skin lid tissue (Fig. 3b) was excised at 24 months (Fig. 3c) to further improve cosmesis at the parents' behest.

$\mathrm{H} \& \mathrm{E}$ sections demonstrated large areas of regressed vessels, characterized by concentric eosinophilic material obliterating the original lumen and interspersed with some residual vessels (Fig. 4a, b). Immunohistochemistry demonstrated collagen IV positivity of the eosinophilic material, confirming it to be basement membrane type material (Fig. 4c). While residual vessels showed marked anti-SMA positivity in their walls, the obliterated vessels showed scanty anti-SMA staining only, indicative of pericyte loss (Fig. 4d). TEM of the effete vessels showed concentric 


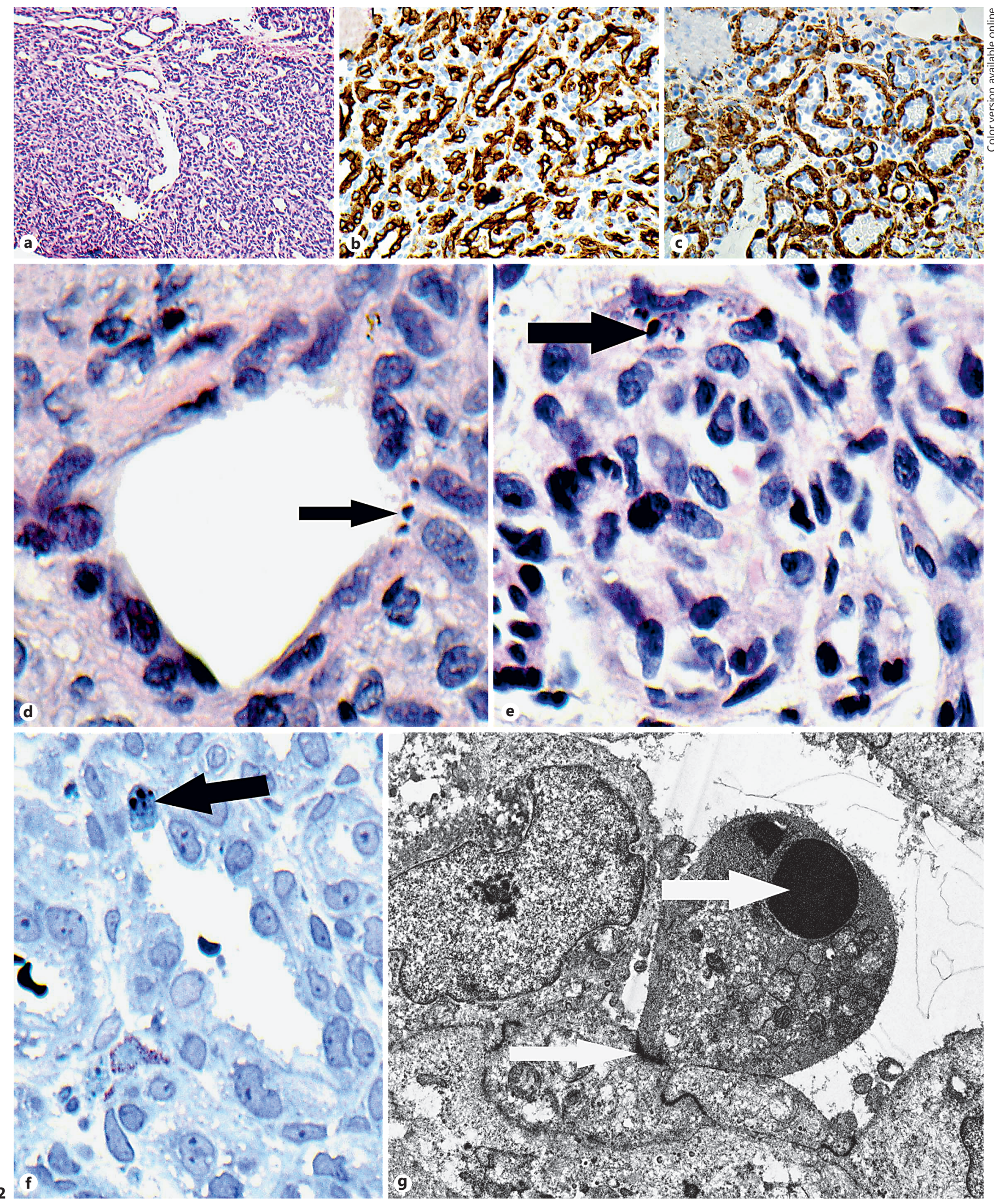

(For legend see next page.)

Ocul Oncol Pathol 2018:4:100-106 DOI: $10.1159 / 00047741$
Sipkova/Xue/Mudhar/Wagner/ Hildebrand 

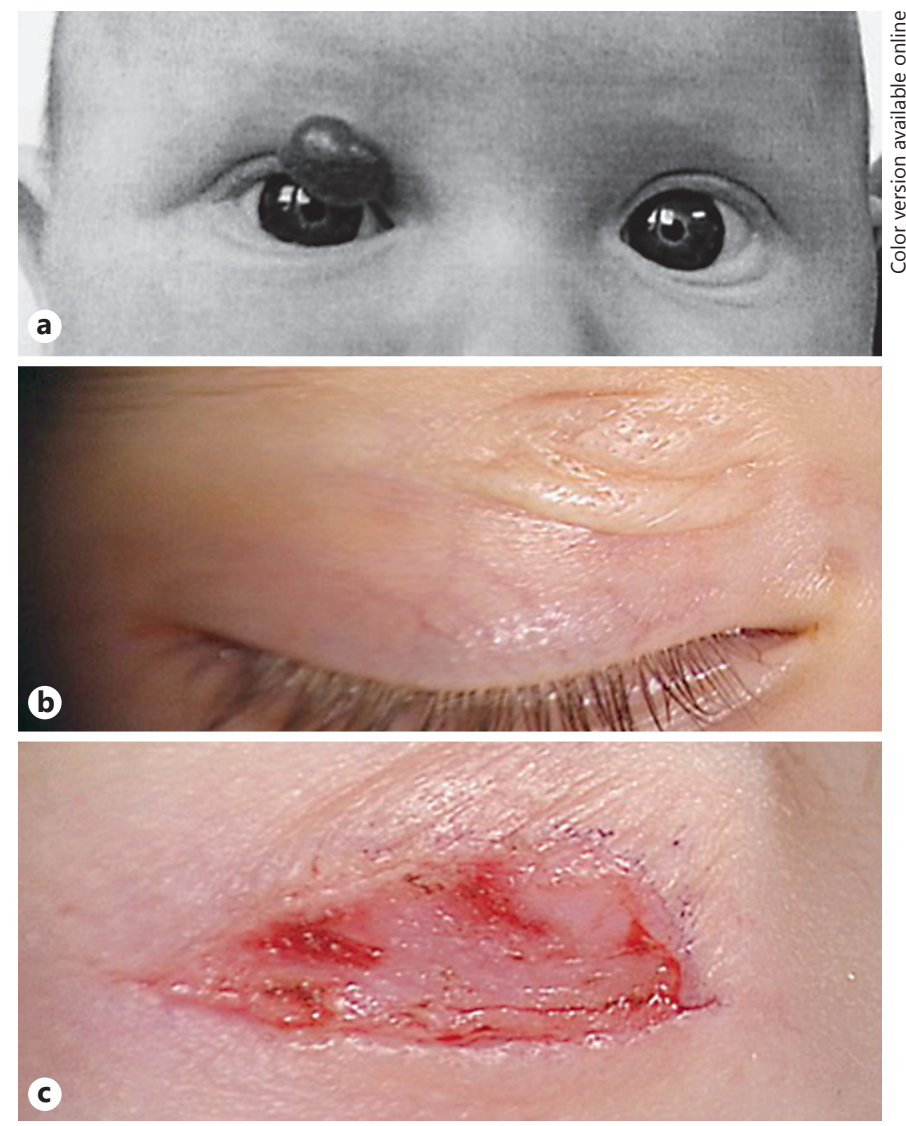

Fig. 3. Photographs of case 2. a A superficial infantile capillary hemangioma of the right upper lid in an 8-month-old girl. b The lesion after 24 months of topical timolol maleate treatment showing excess skin tissue. c Surgical excision of the regressed capillary hemangioma.

Fig. 2. Histopathology and transmission electron micrographs (TEM) of an infantile capillary hemangioma after 1 week of topical timolol maleate treatment (case 1) compared with a spontaneously regressing control. a Hematoxylin and eosin (H\&E) stain showing a capillary hemangioma with crowded, back-to-back capillarysized vessels. b Immunohistochemistry staining with CD31 shows positive endothelial cells. c Immunohistochemistry with antismooth muscle actin shows positive pericytes. d, e H\&E showing apoptotic cells in the endothelial cells (arrows). $\mathbf{f}$ A toluidine bluestained section showing an apoptotic endothelial cell lining the lumen (arrow). g TEM showing an endothelial cell with a tight junction (narrow arrow) with a neighboring viable endothelial cell undergoing apoptosis with a fragmenting electron dense nucleus (thick arrow) compared with the intact nuclei of neighboring endothelial cells. Magnification is $\times 406,000$.

Histopathology of Topical Beta-Blocker-

Treated Capillary Hemangioma layers of basal lamina, containing dystrophic calcium deposits and occasionally surrounded by thin cytoplasmic processes of pericytes (Fig. 4e). No apoptotic bodies were seen. Comparison was made with TEM of spontaneously regressed capillary hemangioma, which showed diffusely packed collagen IV within the interstitial matrix and some fibrillar collagen (Fig. 4f). No concentric collagen IV deposition, pericyte processes or calcific bodies were seen.

\section{Discussion}

Several molecular mechanisms have been hypothesized to account for the effects of $\beta$-adrenergic blockade on capillary hemangiomas, based on existing understanding of the noradrenaline signaling pathways, but with little formal histopathologic evidence [8]. It was thought that an early vasoconstrictive effect, seen as reduced redness and rapid softening of the lesion, was possibly due to reduced nitric oxide synthesis signaled via adenylyl cyclase and cyclic AMP-dependent protein kinase A within the endothelial cells. Intermediate effects on the cessation of hemangioma growth has been attributed to the inhibition of hypoxia-inducible factor (HIF)1 , associated with reduced production of proangiogenic factors such as vascular endothelial growth factor, basic fibroblast growth factor and matrix metalloproteinases [9]. As a long-term effect, $\beta$-blockade was also thought to promote apoptosis by inhibiting endothelial cell survival signaling mediated by the anti-apoptotic tyrosine kinase (SRC).

We here report the first histopathological and electron microscopic evidence of the cellular reactions to topical $\beta$-blockade in vivo at both early (1 week) and late (24 months) time points following the initiation of topical timolol treatment in 2 children. Apoptosis was observed within $\mathrm{IH}$ at 1 week of topical $\beta$-adrenergic inhibition, supporting a prominent role for $\beta$-adrenergic inhibition in the disruption of endothelial cell survival during the proliferative phase of hemangioma growth. This would be consistent with in vitro studies showing that hemangioma-derived endothelial cells express both $\beta_{1^{-}}$and $\beta_{2^{-}}$ adrenergic receptors and propranolol treatment caused increased levels of apoptosis [10]. Similarly, apoptosis was also previously observed in two specimens of IH resected in patients undergoing propranolol treatment [11].

The difference in the rate of clinical response to topical timolol maleate in the 2 cases likely relates to the degree of drug penetration into the different depths of the hemangiomas (deep in case 1 and more superficial in case 2). 

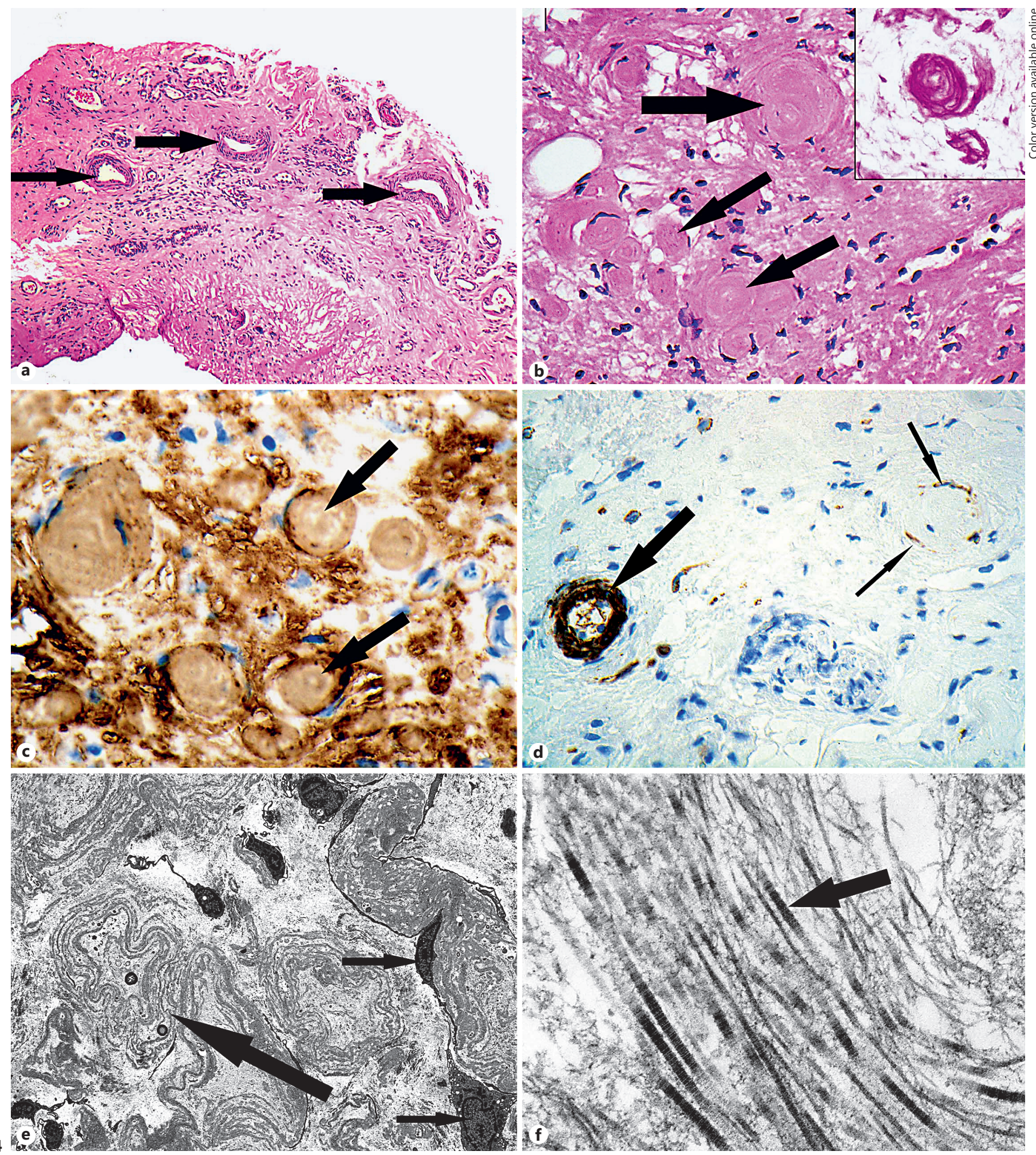

(For legend see next page.)

Ocul Oncol Pathol 2018:4·100-106

DOI: $10.1159 / 00047741$
Sipkova/Xue/Mudhar/Wagner/

Hildebrand 
One might ask why $\beta$-adrenergic inhibition would trigger apoptosis in IH but not in other tissues normally regulated by the $\beta$-adrenergic system, such as the heart and lungs? In cardiomyocytes, noradrenaline derived from the postganglionic sympathetic neurons appears to induce apoptosis through preferential stimulation of (proapoptotic) $\beta_{1}$ - over (antiapoptotic) $\beta_{2}$-adrenoreceptors [12]. In contrast, the lack of sympathetic innervation in IH means that circulatory adrenaline, which stimulates $\beta_{1^{-}}$and $\beta_{2^{-}}$ receptors equally, might normally elicit an antiapoptotic effect overall. Therefore, propranolol might exert its ac-

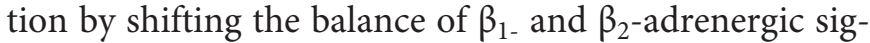
naling within IH towards apoptosis. Another possibility is that the propranolol-induced apoptotic response requires synergistic input from the HIF pathway and is therefore specific to hypoxic tissue. This would be consistent with the expression of GLUT1 by endothelial cells in $\mathrm{IH}$, as it is upregulated by HIF-1 [13]. Interestingly, propranolol has recently been shown to reduce the chance of disease progression in retinopathy of prematurity [14]. The mechanism of action is thought to be analogous to the effect in $\mathrm{IH}$ in that $\beta$-blockade is thought to reduce vascular endothelial growth factor expression (and angiogenesis) within the hypoxic retina by inhibition of HIF-1 and nitric oxide synthase.

When comparing spontaneously regressed IH with $\beta$-antagonist-treated $\mathrm{IH}$, distinct morphological differ-

Fig. 4. Histopathology and transmission electron micrographs (TEMs) of an infantile capillary hemangioma after 24 months of topical timolol maleate treatment (case 2). a H\&E stain of the residual upper lid tissue showing some viable hemangioma vessels (arrows). b H\&E stain of the residual upper lid tissue showing regressed vessels with eosinophilic concentric, variably sized deposited material, assuming the shape and caliber of the original vessel (arrows). The top right inset figure is a periodic acid Schiffstained section showing the deep magenta staining of the concentric deposited material. c Immunohistochemistry with type IV collagen antibody showing variable positivity (brown is positive - arrows) in the eosinophilic material shown in b. d Immunohistochemistry with anti-smooth muscle actin showing strong staining in the pericytes in the wall of the viable patent vessel (thick arrow) and very wispy staining in residual cells around the eosinophilic material in regressed vessels (narrow arrows). e TEM showing concentric type IV collagen deposits in the areas of regressed vessels (thick arrow), surrounded by pericytes with fairly long cytoplasmic processes (thin arrows). Magnification is $\times 73,100$. $\mathbf{f}$ TEM of a spontaneously regressing capillary hemangioma showing fibrillar collagen deposits with periodic banding (arrow). Magnification is $\times 1,930,000$.

Histopathology of Topical Beta-Blocker-

Treated Capillary Hemangioma ences were observed. The former was characterized by diffuse collagen deposition and interstitial fibrosis. In contrast, treated IH showed concentric collagen IV deposition within obliterated vessel lumen, suggestive of waves of endothelial cell apoptosis, leaving behind layers of basement membrane deposits as a stress response. The observation of preserved but scanty pericytes surrounding obliterated vessel lumens in treated $\mathrm{IH}$ would suggest that timolol selectively induced the regression of endothelial cells with relative sparing of pericytes.

Although $\beta$-adrenergic antagonists have shown clinical efficacy in the treatment of IH, their mechanisms of action are still not well understood. These first histopathological and electron microscopic findings of early and late topical $\beta$-blockade provide support for the early induction of endothelial cell apoptosis and endovascular occlusion through concentric deposition of collagen IV as key outcomes of $\beta$-blocker-induced regression of IH. Further understanding of the mechanisms involved in this process will hopefully lead to optimization of the efficacy, dosing and duration of $\beta$-blocker treatment in infantile hemangioma as well as provide new insights into similar other disease processes and open new approaches for their possible treatment with $\beta$-blockers.

\section{Statement of Ethics}

Parental consents were obtained for all procedures as well as permission for the publication of medical photographies.

\section{Disclosure Statement}

The authors declare that they have no conflicts of interest.

\section{Author Contributions}

Study design: G.D.H. Clinical data collection: Z.S., K.X., and G.D.H. Histopathology and electron microscopy: H.S.M. and B.W. Literature search: Z.S., K.X., and G.D.H. Data interpretation: Z.S., K.X., H.S.M., and G.D.H. Preparation, review and approval of manuscript: all co-authors. 


\section{References}

1 Drolet BA, Swanson EA, Frieden IJ: Infantile hemangiomas: an emerging health issue linked to an increased rate of low birth weight infants. J Pediatr 2008;153:712-715.

2 Léauté-Labrèze C, Dumas de la Roque E, Hubiche T, Boralevi F, Thambo JB, Taïeb A: Propranolol for severe hemangiomas of infancy. N Engl J Med 2008;358:2649-2651.

3 Guo S, Ni N: Topical treatment for capillary hemangioma of the eyelid using $\beta$-blocker solution. Arch Ophthalmol 2010;128:255-256.

4 Xue K, Hildebrand GD: Topical timolol maleate $0.5 \%$ for infantile capillary haemangioma of the eyelid. Br J Ophthalmol 2012;96:15361537.

5 Xue K, Hildebrand GD: Deep periocular infantile capillary hemangiomas responding to topical application of timolol maleate, $0.5 \%$, drops. JAMA Ophthalmol 2013;131:12461248 .
6 Painter SL, Hildebrand GD: Review of topical beta blockers as treatment for infantile hemangiomas. Surv Ophthalmol 2016;61:51-58.

7 Painter SL, Hildebrand GD: Topical timolol maleate $0.5 \%$ solution for the management of deep periocular infantile hemangiomas. J AAPOS 2016;20:172-174.

8 Storch $\mathrm{CH}$, Hoeger PH: Propranolol for infantile haemangiomas: insights into the molecular mechanisms of action. Br J Dermatol 2010;163:269-274.

9 Chim H, Armijo BS, Miller E, Gliniak C, Serret MA, Gosain AK: Propranolol induces regression of hemangioma cells through HIF1 alpha-mediated inhibition of VEGF-A. Ann Surg 2012;256:146-156.

10 Stiles J, Amaya C, Pham R, Rowntree RK, Lacaze M, Mulne A, Bischoff J, Kokta V, Boucheron LE, Mitchell DC, Bryan BA: Propranolol treatment of infantile hemangioma endothelial cells: a molecular analysis. Exp Ther Med 2012;4:594-604.
11 Steel R, Day D: Increased apoptosis and secretion of tryptase by mast cells in infantile haemangioma treated with propranolol. Pathology 2014;46:496-500.

12 Rehsia NS, Dhalla NS: Mechanisms of the beneficial effects of beta-adrenoreceptor antagonists in congestive heart failure. Exp Clin Cardiol 2010;15:e86-e95.

13 Xu O, Li X, Qu Y, Liu S, An J, Wang M, Sun Q, Zhang W, Lu X, Pi L, Zhang M, Shen Y: Regulation of glucose transporter protein-1 and vascular endothelial growth factor by hypoxia inducible factor $1 \alpha$ under hypoxic conditions in Hep-2 human cells. Mol Med Rep 2012;6:1418-1422.

14 Filippi L, Dal Monte M, Casini G, Daniotti M, Sereni F, Bagnoli P: Infantile hemangiomas, retinopathy of prematurity and cancer: a common pathogenetic role of the $\beta$-adrenergic system. Med Res Rev 2015;35:619-652. 